\title{
Consumo de contenidos en medios de comunicación por parte de personas mayores en Cataluña
}

\author{
Héctor NAVARRO-GÜERE \\ Universitat de Vic - Universitat Central de Catalunya \\ hector.navarro@uvic.cat \\ Eulàlia MASSANA-MOLERA \\ Universitat de Vic - Universitat Central de Catalunya \\ eulalia.massana@uvic.cat
}

Recibido: 18 de septiembre de 2015

Aceptado: 13 de noviembre de 2015

\begin{abstract}
Resumen
Como adultos de la era predigital, los mayores participan de la ecología mediática en constante evolución donde su participación es diversa. Este trabajo estudia el consumo de medios tradicionales y nuevos medios de los segmentos 39-48, 49-58 y 59-76 años, residentes en Cataluña. Entre las conclusiones destacan que los mayores consumen más prensa escrita. Los dispositivos de acceso a los medios online más usados son los ordenadores de sobremesa, lo consultan en casa y/o en el transporte público; consumen redes sociales, usan la mensajería instantánea y visionan cada vez más vídeos.
\end{abstract}

Palabras clave: Nuevas tecnologías; Televisión; Prensa; Redes Sociales; Teléfonos inteligentes; Mayores.

\section{Content's consumption of Media by Elderly in Catalonian}

\begin{abstract}
As adults from the pre-digital era, senior people participate in an evolving Media Ecology where their contribution is diverse. This paper studies the consumption of Media and New Media by age groups 39-48, 49-58 \& 59-76 years old, residents in Catalonia. One of the most important findings states that elderly people consume more Press. The devices more often used to access the New Media are desktops computer, and usually the access happens at home, and/or traveling by public transport; at the same time they also consume Social Media, use instant messaging and increasingly watch more videos.
\end{abstract}

Keywords: New Media; Television; Press; Social Media; Smartphone; Senior.

\section{Referencia normalizada}

Navarro-Güere, H. y Massana-Molera, E. (2015). Consumo de contenidos en medios de comunicación por parte de personas mayores en Cataluña. Historia y Comunicación Social. Vol 20, número 2, páginas $515-531$. 
Sumario: 1. Introducción. 2. Algunos datos sobre el uso y consumo de tradicionales y nuevos medios por parte de personas mayores. 2.1. Sobre medios tradicionales. 2.2. Sobre nuevos medios. 2.3. Sobre tradicionales y nuevos contenidos. 2.4. Sobre la relación de consumo, preferencias y usos. 3. Objetivo general y metodología. 4. Estudio de campo. 4.1 Consumo de contenidos en televisión. 4.2 Consumo de contenidos en radio. 4.3 Acceso a medios online, dispositivos, lugar de consulta y servicios online consultados. 5. Conclusiones. 6. Referencias bibliográficas. 7. Agradecimientos.

\section{Introducción}

La alfabetización en Tecnologías de la Información y Comunicación (TIC) puede definirse como las destrezas y habilidades que permiten el uso del ordenador y las TIC para desempeñar metas personales, educativas y laborales (Lowe y McAuley, 2000). Entre los temas que trabaja se hallan las implicaciones, frecuencia y contexto de uso del ordenador y otros recursos TIC, el grado de complejidad de las tareas, las barreras, receptividad y desarrollo de habilidades en el sector, y la consideración de los estudios de los adultos con la concepción del llamado "Aprendizaje a lo Largo de la Vida" (Life Long Learning) promovido por la UNESCO (Delors, 1996).

Si nos referimos en la participación de los mayores en la TIC, hallamos aspectos a estudiar como la brecha digital y sociabilidad online (Herrero y Gracia, 2009). Se trata de un término acuñado a mediados de los noventa en los foros donde se discutía si debían o no regularizarse las fuerzas de mercado que estaban surgiendo a raíz de la expansión de las TIC. De forma general se refieren a personas de la era predigital que conviven en una doble realidad y su desenvolvimiento en este no ya tan nuevo escenario es todo un reto (Villanueva, 2006). En este sentido, en su acceso a las TIC se podrían identificar cuatro dimensiones (Van Dick y Hacker, 2003): la motivación para acceder, el acceso material, las competencias para el acceso, y el acceso para los usos avanzados o más sofisticados.

Si nos centramos en la problemática de la brecha digital, esta se refiere al resultado de las brechas sociales producto de la desigualdades económicas, culturales, sociales, políticas, generacionales, geométricas, de género, etc. (Caridad, Ayuso-Sánchez y Ayuso-García, 2010; Contreras-Pulido, Marfil-Carmona y Ortega, 2014); también se tiene que entender como un problema multidimensional (Vega-Almeida, 2007). Es por ello que la brecha digital puede hacer referencia a diversos factores de uso de la tecnología (Massana-Molera. 2012). Existen evidencias que la utilización de las TIC no está al alcance de la totalidad de la población del planeta, ni tan siquiera por la inmensa mayoría de la ciudadanía occidental, no digamos de los jubilados (Pavón-Rabasco, 2000).

Si nos centramos en la sociabilidad online de las personas mayores, se observa que hay herramientas de redes basadas en las TIC, tales como la informática social, que proporcionan nuevas formas de ser social y facilitan la interacción con otras 
personas. Alguna investigación habla que las personas mayores no suelen interesarse en la tecnología como aprendizaje para su propio bien, pero sí cuando el aprendizaje incluye el uso de las TIC se vuelve más relevante (Ala-Mutka, Malanowski, Punie y Cabrera, 2008).

La información constituye un recurso que origina un espacio de contraste entre quienes la tienen y quienes no; a su alrededor se generan simultáneamente oportunidades y desigualdades. Estas desigualdades re- feridas al acceso a la información y al conocimiento mediante las Nuevas Tecnologías que lo facilitan se recogen también bajo el concepto de brecha digital (Robles y Molina, 2007; Prado, 2003). No sólo para los adultos mayores que usan y consumen medios, contenidos y nuevos soportes y plataformas sino también para los responsables de trazar rutas de acción que involucre a este numeroso colectivo. El acceso a las TIC se entiende como herramienta y no como fin en sí mismo ya que son instrumentos que responden a objetivos específicos que tienen que ver con la mejora de las posibilidades de inserción laboral, la necesidad de aprender a lo largo de la vida, de informarse, de comunicarse, de entretenerse y de participar en la comunidad (Flecha y Elboj, 2000).

Figura 1. Uso del teléfono móvil e internet en Catalunya (por género y edad).

Periodo 2010-2013.

\begin{tabular}{|c|c|c|c|c|c|c|c|c|}
\hline & \multicolumn{2}{|c|}{2013} & \multicolumn{2}{|c|}{2012} & \multicolumn{2}{|c|}{2011} & \multicolumn{2}{|c|}{2010} \\
\hline & 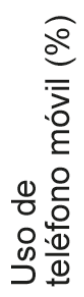 & 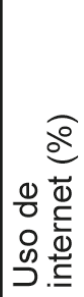 & 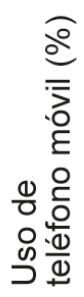 & 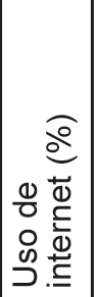 & 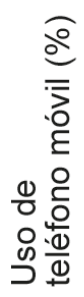 & 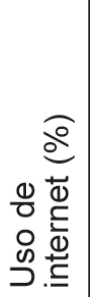 & 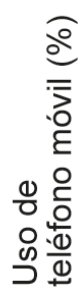 & 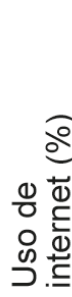 \\
\hline \multicolumn{9}{|l|}{ Género } \\
\hline Hombre & 94,8 & 73,8 & 96,1 & 75,2 & 94,8 & 72,3 & 92,6 & 70 \\
\hline Mujer & 94,4 & 65,2 & 94,8 & 65,8 & 94,1 & 62,7 & 93,6 & 65,3 \\
\hline \multicolumn{9}{|l|}{ Edad } \\
\hline 16-24 años & 98,6 & 99 & 97,5 & 98,5 & 100 & 95,4 & 96,8 & 92,4 \\
\hline 25-34 años & 99,3 & 86,1 & 100 & 87,5 & 100 & 80,9 & 98,3 & 84,9 \\
\hline 35-44 años & 98 & 78,4 & 99,3 & 81,4 & 98 & 79,1 & 97 & 81,5 \\
\hline 45-54 años & 92,7 & 65 & 95,5 & 69,2 & 94,4 & 67,5 & 95 & 65,4 \\
\hline 55-64 años & 92,3 & 51,4 & 91,4 & 45,2 & 86,8 & 42,1 & 87,9 & 40 \\
\hline 65-74 años & 82,1 & 26,3 & 83,5 & 28,3 & 81,9 & 25,3 & 75,1 & 20,7 \\
\hline
\end{tabular}

Fuente: Institut d'Estadística de Catalunya, 2014. 
En la figura 1 se muestra el uso del teléfono móvil e internet en los últimos cuatro años en España, segmentados por género y edad. En la figura no se aprecian diferencias significativas entre géneros aunque no es una batalla ganada: A pesar de los avances legales, sociales, políticos, económicos y laborales que han conseguido las mujeres en los últimos años, todavía persisten importantes desigualdades entre hombres y mujeres (Larrondo, 2005; Navarro-Beltrá, 2009).

En esta línea, alguna investigación ha evaluado cómo las TIC encajan experiencias del trabajo, el ocio y la jubilación (Selwyn, 2004). Hombres y mujeres mayores utilizan con frecuencia ordenadores e internet para el ocio y a veces para actividades de ocio online, como los juegos o navegar por internet. Cubren sus necesidades de información al hacer uso de internet debido a la gran diversidad de fuentes de donde pueden obtener información de temática variada que responde a sus intereses; incluso podrían prescindir de los medios tradicionales ya que saben que se puede acceder a ellos a través de Internet. De hecho, la mayoría lee prensa digital y escucha música en línea, aunque la televisión, siguen utilizando el medio tradicional. También temen el cambio tecnológico porque no son capaces de imaginar el alcance que puede tener debido a la rapidez de los avances y al mismo tiempo no quieren depender de la tecnología. (Álvarez-Arámbula, 2014). Sin embargo, mientras participan en estas actividades, los jubilados son a menudo reticentes a definirlos como una actividad de ocio. Esta reticencia sobre que el ocio vinculado a las TIC puede reflejarse en diferentes segmentos de edad y particularmente como las personas mayores tienen más actitudes de lo que se suele atribuir, contrasta con los estudios con niños que parecen más propensos a adoptar el uso de las Nuevas Tecnologías para el ocio y entretenimiento de forma más natural (Livingstone, 2002).

\section{Algunos datos sobre el uso y consumo de tradicionales y nuevos medios por parte de personas mayores}

No es frecuente encontrar investigaciones que estudien la tercera edad y el consumo de tradicionales y nuevos medios (Baròmetre de la Comunicació i la Cultura, 2011). Por su parte, el informe titulado "Pantalles, continguts i usuaris. Panorama de la convergència mediática digital, els continguts i el consum a Catalunya" (Navarro-Güere, González, Massana-Molera, García, Contreras y Piñero, 2012a) se estudia el fenómeno por segmentos de edad. A continuación se precisan aspectos referidos a las personas adultas y mayores.

\subsection{Sobre medios tradicionales}

En el antes citado estudio, los mayores entrevistados prefieren la televisión y la TDT porque ha significado una ampliación de la oferta. Sobre los contenidos de la televisión, todos prefieren las noticias y las series, y lo hacen por ocio y entretenimiento. Los mayores entrevistados usan internet en el ordenador pero tienen alguna 
dificultad técnica. Para ellos es difícil manejar la jerga técnica. Allí juega un papel importante la asesoría de los hijos y los nietos, aunque hay quien ha hecho algún curso específico. En detalle, las mujeres mayores visionan la televisión como una vía de escape en los momentos de ocio y relajación después del trabajo. La televisión les permite relajarse y desconectar después de un día laboral. Por su parte, los hombres mayores mencionan otros medios tradicionales además de la televisión, concretamente la prensa escrita y la radio. Los entrevistados de este grupo de edad dedican gran parte de su tiempo libre en actividades que involucran a los medios tradicionales, y destacan su uso sobre todo por el hecho de estar informados.

\subsection{Sobre nuevos medios}

Por género, las mujeres mayores entrevistadas en el estudio utilizan el ordenador para temas laborales. Reconocen dedicarle mucho más tiempo que en cualquier otro tema, incluso lo utilizan durante el tiempo de ocio y fines de semana. Otra opina que el ordenador portátil se ha ido incorporando en la vida de forma habitual, aunque con ciertos recelos con respecto a otros medios.

Por su parte, el teléfono móvil es un elemento necesario pero que ha significado para muchos más problemas en su utilización que el uso del ordenador, por ejemplo. Este segmento reconoce utilizarlo sólo para temas familiares, por lo que prescinden de la utilización de teléfonos con mayores prestaciones, como los teléfonos inteligentes, ya que, aunque saben que con este aparato pueden hacer fotos o escuchar música, no lo hacen. Las mujeres mayores entrevistadas reconocen que la principal barrera es la tecnológica y la jerga técnica. En el caso de necesitar utilizar nuevos medios es indispensable la ayuda y orientación de hijos y nietos.

Por su parte, los hombres mayores entrevistados usan las nuevas tecnologías como el ordenador que no sólo utilizan durante el día a día sino también durante las vacaciones, aunque no de igual forma. El teléfono móvil es el dispositivo que este segmento utiliza menos y con el que muestra más recelos en su utilización, limitando su uso a situaciones de emergencia o cuando están fuera de casa. Es una forma de estar conectados pero no manifiestan ningún tipo de dependencia del mismo. Destaca igualmente, el uso del teléfono móvil para escuchar la radio, por ejemplo. Reconocen no ser usuarios de ordenadores, aunque en algún momento se han sentido interesados en aprender su funcionamiento.

\subsection{Sobre tradicionales y nuevos contenidos}

El segmento femenino de la tercera edad entrevistada se decanta por la consulta de noticias, series y telenovelas y prefieren decidir el momento de visionado. En cuanto a los contenidos en los nuevos medios, hay quien menciona que consulta contenidos en internet, sean éstos noticias, películas y series. También hay quien reconoce el uso de redes sociales para estar en contacto con seres queridos y familiares, y la utilización del correo electrónico en el trabajo para mantenerse en contacto, además de acceder a contenidos online por motivos principalmente de ocio. 
Por su parte, los hombres mayores entrevistados reconocen ver la televisión sobre todo para estar informados y entretenerse, como los noticiarios, actualidad.

\subsection{Sobre la relación de consumo, preferencias y usos}

Para las mujeres mayores entrevistadas, el tiempo destinado a la televisión se reduce a momentos puntuales. Se consume más como un método de escape después de la jornada laboral y para sentirse en compañía, o también porque la televisión es una compañía y una parte importante de la rutina diaria. Ante la televisión, varias entrevistadas afirmaron que pueden pasar más de dos o tres horas, en cambio, ante el ordenador menos. Sus hábitos cambian en verano pues salen fuera. El ordenador, por su parte, es utilizado en dos sentidos: por un lado le dedican un tiempo importante durante el día a día laboral (herramienta de trabajo), y por otro lado es un medio para estar conectado con otros; en particular con familiares y amigos, y ocio.

Por su parte, hombres mayores el consumo de nuevos medios sí varía. Al parecer el ordenador lo relacionan más con aspecto laborales mientras que la televisión está vinculada a momentos de esparcimiento, aunque en ocasiones el primero también es utilizado en menor media para la búsqueda de información relacionada con el tiempo libre.

\section{Objetivo general y metodología}

El objetivo principal de este estudio es conocer el consumo de medios por parte de los lectores, radioyentes, usuarios web y televidentes de medios locales, residentes en Cataluña. Para ello, se creó una encuesta online (escala Likert) a mayores de edad, la cual estuvo activa el primer trimestre de 2012. Una vez filtrados los resultados, la muestra útil analizada fue 1.337 encuestas. Los datos mostrados en este trabajo, así como también se hizo en el original, corresponden a las medianas del segmento de edad correspondiente.

Para segmentar la población objeto de estudio se han utilizado las variables sociodemográficas: género, provincias, formación y edades (segmentos analizados: 39-48, 49-58 y 59-76 años, según datos recolectados). La descripción en porcentaje de la muestra según las variables son:

- Por género: mujeres: 48,3\% y hombres: 51,7\%.

- Por segmento de edades: 39-48 años: 56,81\%; $49-58$ años: $32,98 \%$ y 59-76 años: $10,21 \%$.

- Por provincias: Barcelona 65,18\%; Girona: 25,92\%; Lleida: $13,14 \%$ y Tarragona: $5,76 \%$.

- Por formación: no universitaria: $24,87 \%$ y universitaria: $75,13 \%$. 
Hay que destacar que los grupos más jóvenes excluidos de la presente investigación son los que marcan de forma más influyente las tendencias a medio plazo. Los jóvenes se han socializado con los soportes y los contenidos de la comunicación digitales que no existían cuando los segmentos de personas adultas y mayores fueron socializados y que, por tanto, son una parte importante de la población adulta puede haber determinadas barreras de entrada a algunos soportes, como por ejemplo la falta de competencia técnica para su uso.

De igual modo, hay que precisar que en el siguiente análisis, cuando se habla de medios nacionales significa medios que no son de alcance local / comarcal, y cuando se habla de nuevos medios se refiere a medios online, Redes Sociales y aplicaciones (Apps) para dispositivos móviles.

En cuanto al sesgo estadístico, el problema básico inicial para seleccionar una muestra de usuarios en Cataluña que además utilicen internet fue la inexistencia de un marco de muestreo de referencia donde efectuar la selección. Es decir, no existe un directorio mínimamente fiable y completo de las personas que utilizan la Red del que se pueda extraer una muestra aleatoria que permita un muestreo probabilístico proporcionalidad.

Con estas carencias, una muestra estadística representativa de los usuarios de internet generalmente se consigue a través de una investigación probabilística sobre la población total. Esto conlleva un fuerte factor de ineficiencia, dado que todavía no hay una penetración homogénea de internet en el conjunto de la población. En otras palabras, hay desigualdades en el uso de internet en función de la edad, la clase social y el lugar de residencia, cuestión que en los últimos años se ha estado subsanado y ha habido un crecimiento del uso de internet y de los dispositivos móviles (figura 1).

\section{Estudio de campo}

Del procesamiento de la encuesta online sobre el consumo de medios por parte de televidentes, radioyentes, usuarios web y personas que visionan medios locales y/o comarcales con residencia en Cataluña, se extraen los siguientes datos: 
Figura 2. A la izquierda, consumo de medios por grupos de edad y, a la derecha, el consumo por medios tradicionales y online, por grupos de edad.
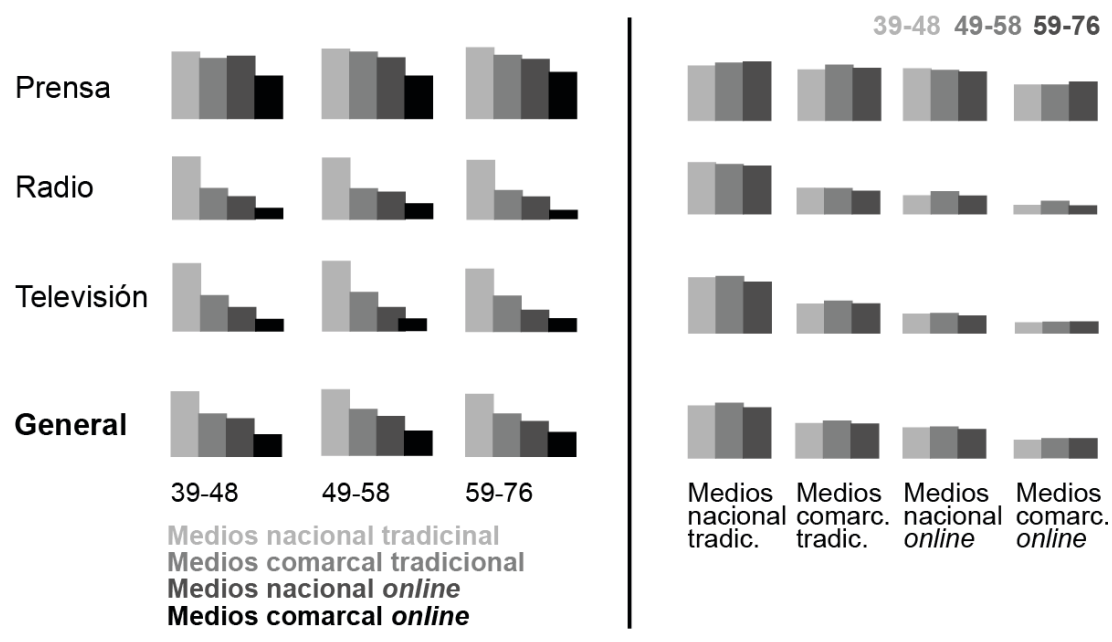

Fuente: elaboración propia.

La primera observación que se desprende de los datos analizados es que el consumo general de medios, en todos los segmentos de edad, sigue un patrón decreciente en este orden: medios nacionales tradicionales (M-NT), comarcales tradicionales (M-CT), nacionales online (M-NO) y comarcales online (M-CO) (figura 2).

Cuando se muestran estos datos segmentados por edades, destaca que también se sigue esta tendencia excepto en la prensa en el segmento de 39-48 años.

Si se analizan los mismos datos pero focalizándonos en la comparativa del consumo a lo largo de las edades (lectura vertical de la figura 2), destaca siempre el consumo superior de los encuestados de 49-58 años respecto a los otros dos. En algunos casos (M-NT y M-NO), el segmento de 39-48 supera en consumo al segmento de 59-76, mientras que en los medios comarcales (tradicionales y online) sucede lo contrario.

En la segmentación por medios (televisión, radio y prensa) se observa que los consumos más elevados, con diferencia, se presentan en los M-NT respecto a los otros formatos (figura 2). Excepcionalmente, el consumo de prensa nacional tradicional (P-NT) no dista mucho de los de prensa comarcal tradicional (P-CT), prensa nacional online $(\mathrm{P}-\mathrm{NO})$ y prensa comarcal online $(\mathrm{P}-\mathrm{CO})$.

A la izquierda de la figura 2 se observa además que no siempre el grupo de edad de 49-58 años es el que consume más; es así en el caso de la televisión nacional tradicional (TV-NT), comarcal tradicional (TV-CT), radio nacional online (R-NO), radio comarcal online ( $\mathrm{R}-\mathrm{CO})$ y prensa comarcal online (P-CO). 
Otro dato a destacar es que el segmento de 59-76 años consume más P-CO que el resto, y también que la P-NT es el formato que es más consumido a medida que aumenta la edad de la muestra analizada.

A continuación se analizarán los contenidos específicos de la televisión y la radio.

\subsection{Consumo de contenidos en televisión}

Figura 3. Consumo de contenidos en los diferentes formatos de televisión y radio, agregados y por grupos de edad.
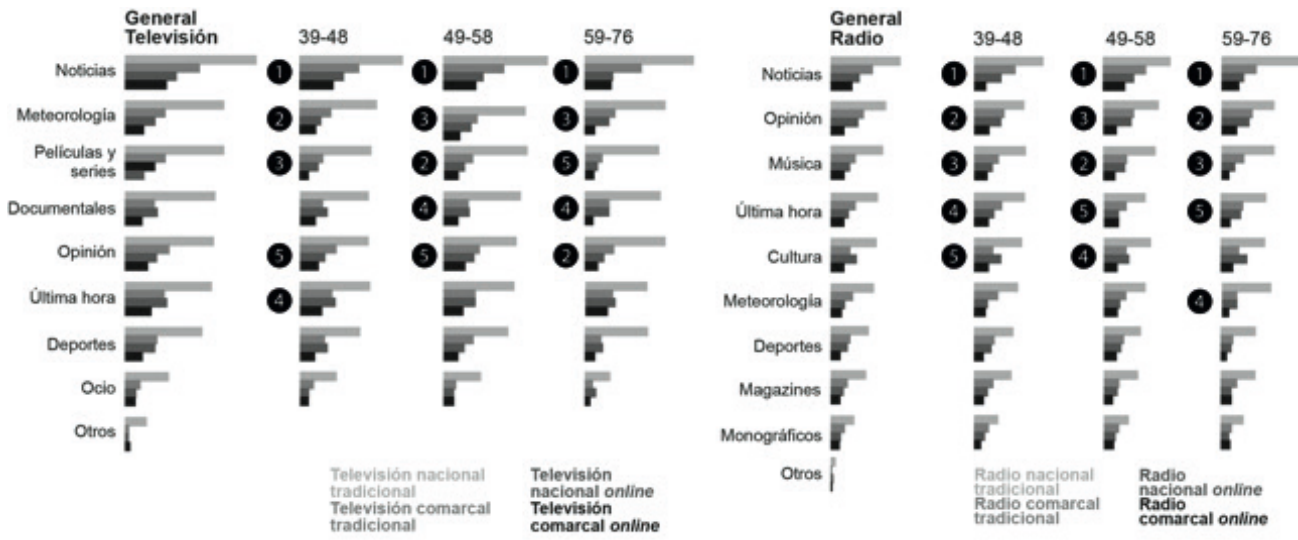

Fuente: elaboración propia.

Los contenidos más consumidos por todos los segmentos de edad en televisión son, sin duda, las "noticias" en medios nacionales (TV-NT) (figura 3). Le siguen la "meteorología", luego "películas y series", en tercer lugar (casi con el mismo valor), "documentales", "opinión" y "última hora".

La relación del consumo de contenidos entre TV-NT, TV-CT, TV-NO, TV-CO siempre es decreciente excepto en "documentales" y "última hora", donde su consumo en TV-NO es superior al de TV-CT.

Si se observan estos mismos datos por grupos de edad (figura 3), los de 39-48 años se nota que el consumo de "última hora" y "documentales" cambian de posición como contenido más consumido. Respecto al medio más consumido, existe la misma tendencia que con el grupo completo, con la particularidad que también el consumo de contenidos de deportes en TV-NO supera al que se realiza en TV-CT.

Por este orden, el segmento de 49-58 años consume: "noticias", "películas y series", "meteorología", "documentales", "opinión", "última hora", "deportes" y "ocio". En este mismo segmento de edad casi se mantiene la tendencia decreciente, en todos los casos, de consumos de TV-NT, TV-CT, TV-NO, TV-CO, y es parecido el consumo de "documentales", "última hora" y "ocio" en TV-MO i TV-CT. 
En el segmento de 59-76 años, el orden de contenidos consumidos es: "noticias", "opinión", "meteorología", "documentales", "películas y series", "deportes" y "última hora". La relación del consumo de contenidos entre TV-NT, TV-CT, TV-NO, TV-CO siempre es decreciente excepto en "noticias" (TV-NO parecido a TV-CT) y en "deportes" y "última hora" (TV-NO por encima de TV-CT).

En la figura 3 se observan los datos desde la perspectiva del consumo de los tres principales contenidos de televisión en los diferentes formatos y por segmento de edad.

El consumo de "noticias" en los más mayores es ligeramente superior sólo en TV-NT, donde los dos primeros segmentos de edad tienen consumos muy parecidos. En el resto de los medios, sigue una tendencia decreciente en TV-NT, TV-CT, TV-NO, TV-CO y en los segmentos de edad, excepto para los dos primeros que también tienen consumos parecidos en TV-CT.

En la figura 3 también se puede observar la perspectiva de análisis desde los grupos de edad. Destaca que el consumo de contenidos de "meteorología" es superior en el segmento de 49-58 años, excepto en TV-CO donde se sigue la tendencia decreciente inversa a la edad. Los valores de los segmentos de 39-48 y 59-76 años son parecidos, pero superiores para los mayores en TV-NT y TV-NO.

El consumo de contenidos de "películas y series" también es superior en el segmento de 49-58 años. Los valores de los segmentos de 39-48 y 59-76 años son parecidos en TV-NT y TV-CO.

Sobre otros contenidos de la televisión hallamos (figura 3):

El consumo de "documentales" es superior para el segmento de 49-58 años sólo en los medios tradicionales. En los medios online es superior en el segmento de 39-48 años. En TV-NT, el consumo de 59-76 años supera al de 39-48 años.

El consumo de contenidos de "opinión" tiene tres comportamientos diferentes según el tipo de medio. En TV-NT, hay un consumo ascendente según aumenta la edad. En TV-CT, los dos primeros segmentos tienen un consumo parecido y es menor en los más mayores. En TV-NO y TV-CO, el grupo intermedio tienen el mayor consumo, luego los de 39-48 años y luego los de 59-76 años.

El consumo de contenidos de "última hora" en TV-NT es parecido para los dos primeros grupos de edad, decreciente según aumenta la edad en TV-CT y TV-NO y menor en el grupo de edad intermedio para TV-CO.

Los "deportes" en TV-NT y TV-CT son consumidos en mayor cantidad por el segmento de 49-58 años. En TV-NO es el grupo de menor edad de la muestra que tiene el consumo mayor.

El consumo de "ocio" sigue el mismo patrón en TV-NT i TV-CO (mayor en 49-58 años, después 39-48 años y después 59-76 años), mientras que es un consumo decreciente (respecto a la edad) en TV-CT y creciente en TV-NO. 


\subsection{Consumo de contenidos en radio}

Según todos los segmentos de edad, los contenidos más consumidos en radio son las "noticias" en medios nacionales (R-NT) (Figura 3). No es tanta la distancia que se nota en los diferentes formatos de radio analizados respecto al que se ha mostrado en televisión (subapartado anterior). Le siguen "música", "opinión", "última hora", "cultura", "meteorología", "deportes", "magazines" y, finalmente, "monográficos".

La relación del consumo de contenidos entre R-NT, R-CT, R-NO, R-CO siempre es decreciente excepto donde en R-NO donde es superior al de R-CT. Como se puede observar en la figura 3 por segmentos de edad, esta tendencia se mantiene en todos ellos. En el análisis del consumo de la televisión, esto sucedía para "documentales" y "última hora".

Si se observan estos mismos datos segmentados por edad (figura 3 ), en el segmento de 39-48 años se observa que el orden del consumo (de más a menos) es: "noticias", "opinión", "música", "última hora", "cultura", "meteorología”, "deportes", "magazines" y "monográficos". En el segmento de 49-58 años se consume, por este orden: "noticias", "música", "opinión", "cultura", "última hora", "meteorología”, "deportes", "magazines" y "monográficos". En el segmento de 59-76 años, el orden de contenidos consumidos es: "noticias", "opinión", "meteorología", "documentales", "películas y series", "deportes" y "última hora".

El consumo de "noticias" en los más mayores es ligeramente superior sólo en R-NT, donde los dos primeros segmentos de edad tienen consumos muy parecidos. En el resto de los medios sigue la misma tendencia donde el orden del consumo de mayor a menor es del segmento de 49-58 años, luego el de 39-48 años y finalmente el de 59-76 años. En el consumo de "noticias" es donde se evidencia una mayor diferencia entre R-NT y el resto de medios (siempre en orden decreciente: R-CT, R-NO y R-CO).

En el segmento de 49-58 años, el consumo de contenidos de "música" es superior en R-NO e inferior en R-CO. En el resto de medios sigue la tendencia decreciente según la edad.

El consumo de contenidos de "opinión" es parecido para R-NT en los tres segmentos de edad: superior para el segmento de 49-58 años en R-NO y decreciente para el resto, según aumenta la edad.

Si se observa el consumo del resto de contenidos de radio en los diferentes formatos por grupo de edad (figura 3 ), se nota que:

El consumo de contenidos de "última hora" es superior para el segmento de 59-76 años en R-NT i R-NO. En R-CO los dos primeros segmentos de edad tienen un consumo parecido: mientras que en R-NT, R-CT y R-NO el segmento de 39-48 años tienen un consumo mayor que el de 49-58 años. 
El consumo de contenidos de "cultura" en R-NO supera al de R-CT, donde además el segmento de edad 59-76 años supera al consumo de 49-58 años. El segmento de edad intermedio sólo supera en consumo de "cultura" en R-CT.

Del consumo de contenidos de "meteorología" destaca por no repetirse en otros casos con esta diferencia el consumo de 59-76 años en R-NT. En R-CT se sigue la tendencia decreciente según avanza la edad y en los formatos online quien consume más es los de 49-58 años. El menor consumo es para R-CO entre el segmento de los mayores.

Los "deportes" son consumidos de manera decreciente según aumenta la edad por R-NT, R-CT, R-NO y R-CO.

El consumo de "magazines" es decreciente según la edad para R-NT y R-CT, mientras que el segmento de 49-58 años consumen más R-NO y menos R-CO. En estos últimos, el mayor consumo es el segmento de edad 59-76 años.

El consumo de los "monográficos" sigue una dinámica parecida al de los "deportes" (decreciente según aumenta la edad), excepto para R-CO donde se invierten estos valores.

4.3. Acceso a medios online, dispositivos, lugar de consulta y servicios online consultados

Figura 4. Dispositivos de acceso para el consumo de medios online. medios nacionales online y medios comarcales online (por segmentos de edad y dispositivo, y viceversa)

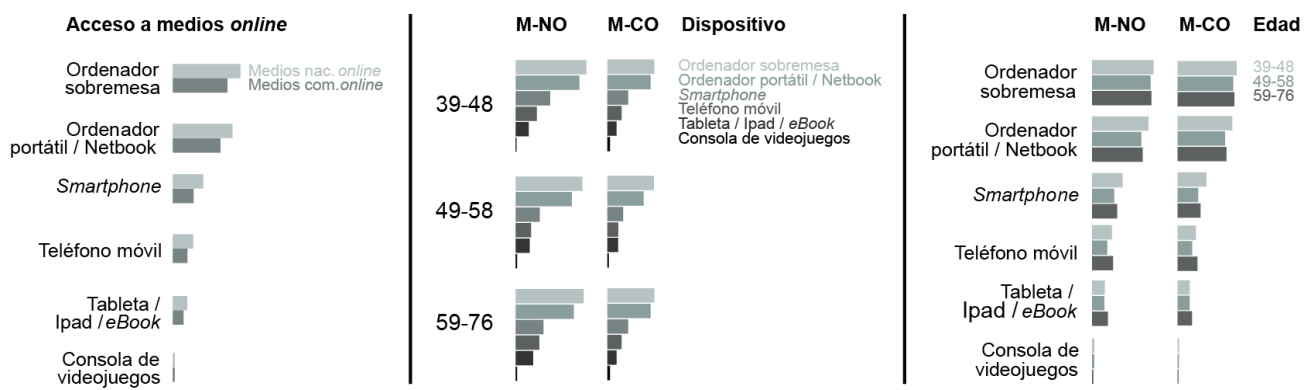

Fuente: elaboración propia.

Los dispositivos de acceso a los M-NO y M-CO son, por orden de uso: "ordenador sobremesa", "ordenador portátil / netbook", "smartphone", "teléfono móvil", "tableta / ipad / lector de libros digitales" y "consola de videojuegos" (figura 4). Siempre el uso es mayor en M-NO que en M-CO.

Si se observa esta distribución para el acceso a M-NO por edades, el orden es el mismo que en el grupo completo (figura 4). En cambio, si se observa el uso de dispo- 
sitivos móviles para acceder a M-NO, se nota que el segmento de edad de 59-76 años los usa más que el de 49-58 años.

Si se observa esta distribución para el acceso a M-CO por edades, el orden es el mismo que en el grupo completo, excepto para el grupo de 49-58 años, donde la "tableta..." supera ligeramente el "teléfono móvil" (figura 4).

El uso dispositivos para acceder a M-NO para el segmento de edad de 59-76 años es mayor que el de 49-58 años en casi todas las opciones.

Figura 5. Desde dónde son consultados los medios online (por segmento de edad y lugar de consulta), y uso de servicios online (segmento de edad y servicio).

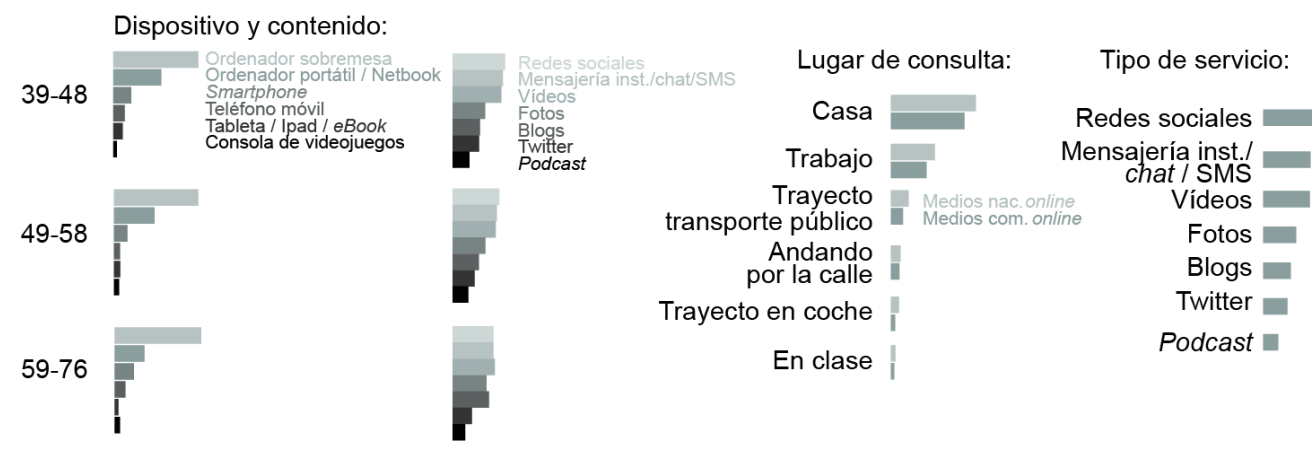

Fuente: elaboración propia.

El orden de consulta de los medios online se mantiene igual en los tres segmentos de edad (figura 5). De mayor a menor, los medios online se consultan desde: "casa", "trabajo", "trayecto transporte público", "andando por la calle", "trayecto en coche" y "en clase".

El uso de servicios, segmentado por edades, no es homogéneo (figura 5). En los segmentos de 39-48 años y 49-58 años es parecido, pero en los más mayores, desciende levemente el uso de "redes sociales" y "mensajería instantánea" para aumentar los "vídeos" y los "blogs". El orden del uso de servicios online es: "redes sociales", "mensajería instantánea / chat / SMS", "vídeos", "fotos", "blogs", "Twitter" y "podcast".

\section{Conclusiones}

Partiendo de la premisa que el acceso a la Sociedad de la Información en personas mayores supone un recurso de sociabilidad de gran relevancia que permite mantener y ampliar la red social más próxima, debe implementarse mejoras en su acceso por parte de todos los actores de la sociedad (Pavón-Rabasco, 2000). Existe cierta 
conciencia que con el paso de la edad, los cambios tecnológicos generan incertidumbres ya que alteran hábitos y estructuras cognitivas (Ala-Mutka, Malanowski, Punie y Cabrera, 2008). En esta línea, partiendo de la muestra analizada destaca que:

El orden de consumo de medios es, en general: Medios nacionales tradicionales, medios comarcales tradicionales, medios nacionales online y medios comarcales online, siendo el consumo de medios nacionales tradicionales muy superior a los otros.

En general, el consumo de medios desciende a medida que asciende la edad, aunque en algunos casos el consumo del segmento intermedio (49-58 años) supera a los otros dos. Aún así, no existe una desconexión total en los más mayores.

En todos los segmentos de edad, el consumo de prensa comarcal tradicional, prensa nacional online y prensa comarcal online es superior a los correspondientes consumos de televisión y radio.

El orden de consumo de contenidos en televisión es "noticias", "meteorología", "películas y series", "documentales", "opinión" y "última hora". Hay algunas diferencias según el segmento de edad.

El orden de consumo de contenidos en radio es: "noticias", "música", "opinión", "última hora", "cultura", "meteorología", "deportes", "magazines" y, finalmente, "monográficos". Hay algunas diferencias según el segmento de edad.

Los dispositivos de acceso a los medios nacionales online y medios comarcales online son, por orden de uso: "ordenador sobremesa", "ordenador portátil / netbook", "smartphone", "teléfono móvil", "tableta/ipad/lector de libros digitales" y "consola de videojuegos". Siempre el uso es mayor en medios nacionales online que en medios comarcales online. Hay algunas diferencias según el segmento de edad.

El orden de la ubicación de consulta de los medios online es: "casa", "trabajo", "trayecto transporte público", "andando por la calle", "trayecto en coche" y "en clase", siendo los dos primeros muy superiores a los otros tres. No hay diferencias según el segmento de edad.

El orden del uso de servicios online es: "redes sociales", "mensajería instantánea/ chat/SMS", "vídeos", "fotos", "blogs", "Twitter" y "podcast". Entre más mayores, desciende levemente el uso de "redes sociales" y "mensajería instantánea" y aumentan los "vídeos" y los "blogs". 


\section{Referencias bibliográficas}

ALA-MUTKA, K.; MALANOWSKI, N.; PUNIE, Y.; CABRERA, M. (2008). Active Ageing and the Potential of ICT for Learning. Sevilla: European Commission; Joint Research Centre, Institute for Prospective Technological Studies. DOI: $10.2791 / 33182$

CONTRERAS-PULIDO, P.; MARFIL-CARMONA, R.y ORTEGA, J. (2014). "La competencia mediática de las personas mayores andaluzas: retos para una inclusión social plena”. En: Historia y Comunicacion Social, Vol. 19, Enero, (Número especial), p.129-142: DOI: 10.5209/rev_HICS.2014.v19.44946

FLECHA, R.; ELBOJ, C. (2000). "La Educación para personas Adultas en la Sociedad de la Información". En: Educación XXI, Vol. 3, p. 141-162. DOI: 10.5944/ educxx1.3.1.407

LIVINGSTONE, S. (2002). Young People and New Media. Londres: Sage.

SELWYN, N. (2004). "The Information Aged: A Qualitative Study of Older Adults' Use of Information and Communications Technology". En: Journal of Ageing Studies 18 (4), p. 369-384. DOI: 10.1016/j.jaging.2004.06.008

VAN DICK, J.; HACKER, K. (2003). "The digital divide as a complex and dynamic phenomenon". En: The Informacion Society, 19, p. 315-326: DOI: $10.1080 / 01972240309487$

\subsection{Referencias web}

ÁLVAREZ-ARÁMBULA, E. (2014). "Usos y gratificaciones en el consumo de las aplicaciones de internet en personas mayores. Un estudio desde la perspectiva de género" (tesis doctoral). Departamento de Comunicación Audiovisual y Publicidad I. Universitat Autònoma de Barcelona, Bellaterra: http://www.tesisenred.net/ handle/10803/284345 [Consulta: 05/07/2015]

BARÒMETRE DE LA COMUNICACIÓ I LA CULTURA (2011). "Els diaris en catalá avui: el nou escenari. De la realitat a la dada, de la dada al coneixement". Barcelona: Fundacc: http://www.fundacc.org/docroot/fundacc/pdf/resum_public_4a_onada11_barometre_cat.pdf [Consulta: 21/10/2014]

CARIDĀD, M.; AYYUSO-SÁN̄CHEZ, M.L.: AYUSO-GARCÍA, M.D. (2010). "Estudio comparado de la brecha digital en los países de la Unión Europea y en España (2004-2008)". Brasilia: Inc. Soc. Vol. 3, nº 2, p. 54-68: http://revista.ibict.br/inclusao/index.php/inclusao/article/viewFile/136/159 [Consulta: 05/07/2015]

DELORS, J. (ed) (1996). La educación encierra un tesoro. Informe a la UNESCO de la Comisión Internacional sobre la Educación para el siglo XXI. Madrid, Ediciones UNESCO: http://unesdoc.unesco.org/images/0010/001095/109590so. pdf [Consulta: 05/07/2015]

INSTITUT D'ESTADÍSTICA DE CATALUNYA (2014). Consulta de datos: http:// www.idescat.cat/pub/?id=ticl113\&n=1.2.1\&lang=es [Consulta: 03/11/2014]

LARRONDO, A. (2005). "La red al servicio de las mujeres. Aproximación a la relación mujer y medios de comunicación en Internet". En: Estudios sobre el mensaje 
periodistico, $\mathrm{n}^{\mathrm{o}} 11$, p. 375-392: http://pendientedemigracion.ucm.es/info/emp/ Numer_11/Sum/4-09.pdf [Consulta: 05/07/2015]

LOWE, G.; McAULEY, J. (2000). Adult Literacy and Lifeskills Survey Information and Communication Technology Literacy Assessment Framework. ALL Survey - ICTL Assessment Framework: http://www.ets.org/Media/Research/pdf/ICTL FRAMEWORK.pdf [Consulta: 05/07/2015]

NAVARRO-BELTRÁ, M. (2009). "La brecha digital de género en España: cambios y persistencias". En: Feminismo/s, no 14 (diciembre), p. 183-200: http://rua.ua.es/ dspace/bitstream/10045/13307/1/Feminismos 14 11.pdf [Consulta: 03/07/2015]

NAVARRO-GÜERE, H.; GONZÁLEZ, Z.; MASS̄ANĀA-MOLERA, E.; GARCÍA, I.; CONTRERAS, R.; PIÑERO, J.C. (2012a) Pantalles, continguts $i$ usuaris. Panorama de la convergència mediática digital, els continguts i el consum a Catalunya. Barcelona, Grup de Recerca d'Interaccions Digitals (Universitat de Vic) y Consell de l'Audiovisual de Catalunya: https://www.cac.cat/pfw_files/cma/ recerca/estudis_recerca/Consum_multipantalla.pdf [Consulta: 03/07/20̄15]

NAVARRO-GÜEREE, H.; CONTRERAS, R.; GARCÍA, I.; MASSANA-MOLERA, E.; GONZÁLEZ, Z. (2012b). La premsa comarcal i el consum de noves pantalles. Vic, Grup de Recerca d'Interaccions Digitals (Universitat de Vic) y Associació Catalana de la Premsa Comarcal: http://www.premsacomarcal.cat/documents/ premi-recerca-vi.pdf [Consulta: 03/07/2015]

MASSANA-MOLERA, E. (2012). "La bretxa generacional en el'ùs de mòbils a Catalunya". En: Obra digital, 2 (febrero), p. 46-56: http://www.raco.cat/index. $\mathrm{php} /$ ObraDigital/article/view/252007/338322 [Consulta: 05/07/2015]

PAVÓN-RABASCO, F. (2000). "Tecnologías avanzadas: nuevos retos de comunicación para los mayores". En: Comunicar, 15, p. 133-139: http://rabida.uhu. es/dspace/bitstream/handle/10272/944/b15205319.pdf?sequence $=1$ [Consulta: 05/07/2015]

PRADO, E. (2003). "La brecha digital o el peligro de exclusión de la Sociedad de la Información". En Quaderns del CAC, no 15 (enero-abril), p. 3-12: http:// www.cac.cat/pfw_files/cma/recerca/quaderns_cac/Q15prado_ES.pdf [Consulta: 05/07/2015]

ROBLES, J.M.; MOLINA, Ó. (2007). "La Brecha digital: ¿una consecuencia más de las desigualdades sociales? Un análisis de caso para Andalucía". En: Empiria, $\mathrm{n}^{\circ} 13$, p. 81-99: http://dialnet.unirioja.es/servlet/articulo?codigo=2330066 [Consulta: 03/05/2015]

VEGA-ALMEIDA, R.L. (2007). "Brecha digital: un problema multidimensional de la sociedad emergente". En: Inclusao Social, Vol. 2, no 2, p. 96-108 http:// revista.ibict.br/inclusao/index.php/inclusao/article/viewFile/52/76: [Consulta: 06/07/2015]

VILLANUEVA, Eduardo (2006). "Brecha Digital: Descartando un término equívoco". En: Razón y Palabra, Vol. 11, no 51: http://www.redalyc.org/articulo. oa? id=199520723003 [Consulta: 05/07/2015] 


\section{Agradecimientos}

Los datos del estudio de campo se han extraídos de la base de datos original del informe "La premsa comarcal i el consum de noves pantalles" (Navarro-Güere, H.; Contreras, R., García, I., Massana-Molera, E., González, Z., 2012b), que contó con la financiación de la Associació Catalana de la Premsa Comarcal. 